\title{
Identification and characterization of CircRNAs involved in the regulation of wheat root length
}

Yanhua $\mathrm{Xu}^{1,2,3,4^{+}}$, Yongzhe Ren ${ }^{1,2,3^{*}+}$ (D), Tongbao Lin ${ }^{1,2,3}$ and Dangqun Cui ${ }^{1,2,3^{*}}$

\begin{abstract}
Background: Recent studies indicate that circular RNAs (circRNAs) may play important roles in the regulation of plant growth and development. Plant roots are the main organs of nutrient and water uptake. However, whether circRNAs involved in the regulation of plant root growth remains to be elucidated.
\end{abstract}

Methods: LH9, XN979 and YN29 are three Chinese wheat varieties with contrasting root lengths. Here, the root circRNA expression profiles of LH9, XN979 and YN29 were examined by using high-throughput sequencing technology.

Results: Thirty-three and twenty-two differentially expressed circRNAs (DECs) were identified in the YN29-LH9 comparison and YN29-XN979 comparison, respectively. Among them, ten DECs coexisted in both comparisons. As the roots of both LH9 and XN979 were significantly larger and deeper than YN29, the ten DECs coexisting in the two comparisons were highly likely to be involved in the regulation of wheat root length. Moreover, three of the ten DECs have potential miRNA binding sites. Real-time PCR analysis showed that the expression levels of the potential binding miRNAs exhibited significant differences between the long root plants and the short root plants.

Conclusions: The expression levels of some circRNAs exhibited significant differences in wheat varieties with contrasting root phenotypes. Ten DECs involved in the regulation of wheat root length were successfully identified in which three of them have potential miRNAs binding sites. The expression levels of putative circRNA-binding miRNAs were correlated with their corresponding circRNAs. Our results provide new clues for studying the potential roles of circRNAs in the regulation of wheat root length.

Keywords: Triticum aestivum L., Root length, CircRNAs, Regulation

\section{Background}

Plant roots are the main organs of nutrient and water uptake from soil. Genetic improvement of root traits is of vital importance for improving crop nutrient and water use efficiencies [1-5]. Understanding the molecular mechanisms controlling plant root growth and development is beneficial to molecular breeding aimed at improving root traits and resource utilization efficiency

\footnotetext{
*Correspondence: yongzheren66@163.com; dangquncui@163.com

${ }^{\dagger}$ Yanhua Xu and Yongzhe Ren contributed equally to this work

${ }^{1}$ College of Agronomy, Henan Agricultural University,

Zhengzhou 450002, China

${ }^{3}$ Collaborative Innovation Center of Henan Grain Crops, Henan

Agricultural University, Zhengzhou 450002, China

Full list of author information is available at the end of the article
}

of crops. Previous studies have shown that plant root growth and development are controlled by phytohormones and sustained by the root apical meristem (RAM) [6-12]. Besides, redox regulation and the balance of ROS also have important roles in maintaining RAM activity [13-15]. Therefore, the growth and development of plant roots are regulated by complex hormone signals and other related pathways.

CircRNAs are endogenous non-coding RNAs produced by back-splicing of pre-mRNA [16]. The $5^{\prime}$ and $3^{\prime}$ ends of the circRNA are linked together to form a covalent closed loop structure [17]. Currently, circRNAs have been widely studied in animals [18-21]. However, the role of circRNA in plants has not attracted enough attention [22]. Until recent years, several literatures 
reported circRNAs identified in plants such as Arabidopsis [23-27], soybean [28, 29], rice [25, 30], maize [31-33], tomato [34, 35], barley [36], tea [37], cotton $[38,39]$ and wheat $[40,41]$. For example, Ye et al. performed genome-wide identification of circRNAs in rice and Arabidopsis using available public RNA-Seq data and identified 12,037 and 6012 circRNAs, respectively. Moreover, the parent genes of over 700 exonic circRNAs were orthologues between rice and Arabidopsis, suggesting the conservation of circRNAs in plants [25]. Chen et al. performed circRNA-Seq on maize seedling leaves and uncovered 2804 circRNAs. They found that sequences related to LINE1-like elements (LLEs) and their reverse complementary pairs (LLERCPs) are significantly enriched in the flanking regions of circRNAs [31]. Furthermore, genes with LLERCP-mediated circRNAs are enriched among loci that are associated with phenotypic variation. Therefore, circRNAs are likely to be involved in the modulation of phenotypic variation by LLERCPs [31]. Moreover, studies showed that circRNAs may also play roles in response to Verticillium wilt in cotton [39], maize iranian mosaic virus infection in maize [32], TYLCV infection in tomato [35], drought stress and nitrogen deficiency in wheat $[38,41]$. These findings indicate that circRNAs are present in different plant species and may play important roles in the regulation of growth and development, and stress response.

Wheat (Triticum aestivum L.) is one of the most important food crops in the world. In wheat, circRNAs have been shown to be involved in response to drought stress. Wang et al. isolated 88 circRNAs and found that 62 circRNAs were differentially expressed under drought stress conditions compared with control [38]. In a recent study, Ren et al. identified six circRNAs involved in the common response to nitrogen deficiency stress and 23 circRNAs involved in the regulation of low nitrogen-promoted root growth in wheat [41]. These studies show that circRNAs may play important roles in responding to abiotic stresses. However, it is unclear whether circRNAs are involved in the regulation of wheat root growth under normal growth conditions. To explore this question, the root circRNA expression profiles of three wheat varieties (including two long root varieties and one short root variety) were obtained using high-throughput sequencing technology. Differentially expressed circRNAs (DECs) were identified and further validated using real-time PCR technology. Target miRNAs of the DECs were predicted and the expression levels of these miRNAs in the roots of LH9, XN979 and YN29 were also examined. This is the first report on the identification of differentially expressed circRNAs in wheat varieties with contrasting root length. The results are helpful for further investigate of the potential roles of circRNAs in regulating wheat root length.

\section{Methods \\ Plant materials}

XN979, LH9 and YN29 are three Chinese wheat varieties. The roots of LH9 and XN979 are significantly longer than those of YN29. Here, XN979, LH9 and YN29 were selected as materials to identify circRNAs involved in the regulation of wheat root length.

\section{Plant growth conditions and evaluation of root phenotype}

Seed sterilization, germination and the growth conditions of wheat plants were conducted as previously described by Ren et al. [42]. Plants were randomly placed and grown in a greenhouse with six replications each. The maximum root length (MRL) and total root length (TRL) of XN979, LH9 and YN29 were analyzed by using WinRHIZO software (Regent Instruments, Canada) after 15 days of transferring. The developmental stages of wheat plants were Zadoks growth scale 13 [43]. The roots of XN979, LH9 and YN29 were fast-frozen using liquid nitrogen for RNA extraction.

\section{Libraries construction and sequencing}

Root total RNA was extracted using Trizol reagent following the manufacturer's procedure. The concentration and purity of total RNA were measured by NanoDrop ND-1000 spectrophotometer (NanoDrop Technologies, Wilmington, DE, USA). Approximately $10 \mu \mathrm{g}$ total RNA was used to deplete the ribosomal RNA (rRNA) following the instructions of the Epicentre Ribo-Zero Gold Kit (Illumina, San Diego, USA). The remaining RNAs were used as templates for the construction of cDNA libraries following the instructions of the RNA-Seq sample preparation kit (Illumina, San Diego, USA) [41]. Three biological replicates were analyzed. The samples were named as XN979-1, -2, -3; LH9-1, -2, -3; YN29-1, -2, -3, respectively. Paired-end sequencing was performed on an Illumina Hiseq 2500 platform (Hangzhou Shangyi biotechnology company, Hangzhou, China).

\section{Identification of circRNAs}

The obtained clean reads were tempted to align with wheat reference genome (Triticum aestivum TGACv1.0) using the bowtie2 (bowtie2-2.2.2) alignment method. The reads of the linear RNA can be mapped appropriately to wheat reference genome, while the reads at the loop forming junctions of circRNAs cannot be directly aligned to the wheat reference genome [41]. Then the find_circ software was employed to detect head-to-tail 
splicing (back-spliced) of the remaining unmapped RNAseq reads (default setting). The detected back-spliced reads were further filtered to predict circRNAs following the recommended setting rules (GU/AG appears on both sides of the splice site, clear breakpoint can be detected; $\leq 2$ mismatches; The length of the circRNA junctions $\leq 100 \mathrm{~kb})[41,44]$.

\section{Differential expression analysis of circRNAs}

Differential expression analysis of circRNAs between different varieties was performed using the DEseq $R$ package [41]. Only the circRNAs with $p$ values $\leq 0.05$ and $\mid \log 2$ (foldchange) $\mid \geq 1$ were defined as DECs $[45,46]$. psRNATarget software was used to predict circRNAmiRNA interactions of the DECs [47].

\section{Real-time PCR analysis}

To confirm and quantify the predicted circRNAs, divergent primers of five randomly selected DECs were designed based on the flanking sequences of the headto-tail splicing site of each circRNA (Additional file 1: Table S1). The cDNA samples were used as templates and mixed with primers and SYBR Green PCR Real Master Mix (Tiangen, China) for real-time PCR analyses. In the quantitative analysis of selected miRNAs, miRcute Plus miRNA First-Strand cDNA Synthesis Kit (Tiangen, China) was used for cDNA synthesis and miRcute Plus miRNA qPCR Detection Kit (Tiangen) was used for quantitative analysis according to the kit instructions. The reverse primers were provided in the miRcute Plus miRNA qPCR Detection Kit and the forward ones were designed according to the instructions of the kit (Additional file 1: Table S1). Real-time PCRs were performed on a Thermal Cycler CFX96 Real-Time System (BioRad, USA). The program settings are as follows: $95{ }^{\circ} \mathrm{C}$ for $5 \mathrm{~min}$, then 40 cycles of $95^{\circ} \mathrm{C}$ for $15 \mathrm{~s}, 60^{\circ} \mathrm{C}$ for $15 \mathrm{~s}$, $72{ }^{\circ} \mathrm{C}$ for $15 \mathrm{~s}$. TaActin was used as an internal reference gene to normalize the expression levels of the investigated DECs and miRNAs. SPSS 21.0 software was used to analyze the statistical significance of the data.

\section{Results}

\section{Root phenotypes of different wheat varieties}

The root phenotypes of LH9, XN979 and YN29 are shown in Fig. 1. There are significant differences between the long root varieties (LH9, XN979) and the short root variety (YN29). The MRL of LH9 and XN979 were $13.7 \mathrm{~cm}$ and $9.2 \mathrm{~cm}$ longer than that of YN29 (Fig. 1a), and the TRL of LH9 and XN979 were $302.4 \mathrm{~cm}$ and $211.9 \mathrm{~cm}$ longer than that of YN29 respectively (Fig. 1b). Overall, the roots of LH9 and XN979 were significantly larger and deeper than those of YN29 (Fig. 1c).

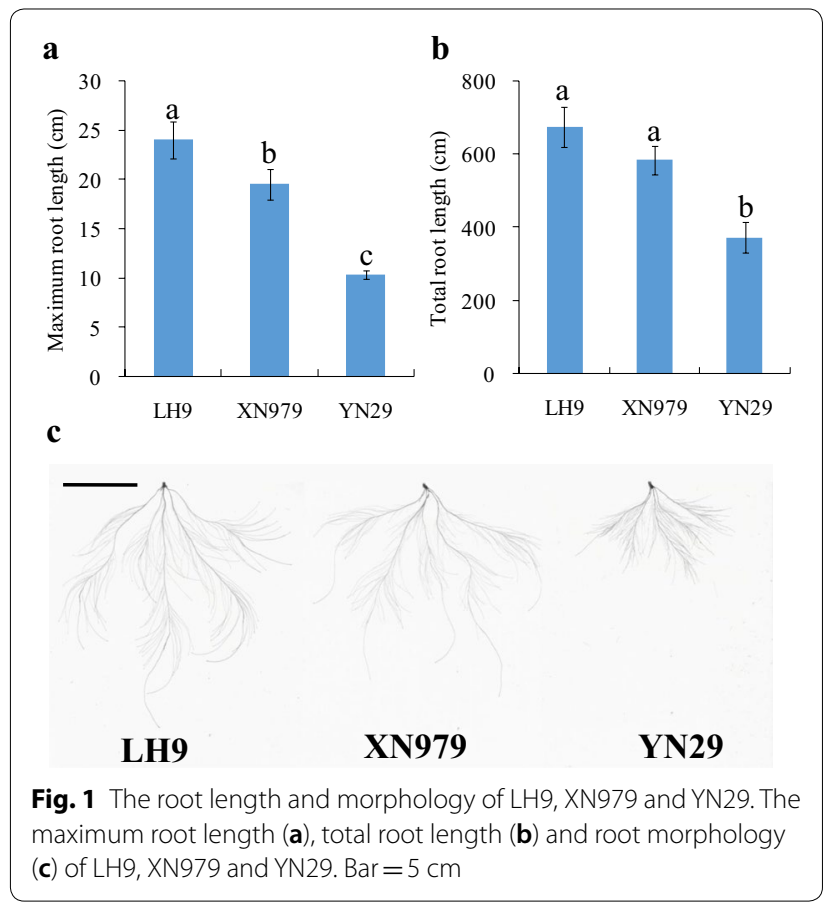

\section{CircRNAs identification}

To determine circRNAs involved in the regulation of root length, nine cDNA libraries from wheat roots of LH9, XN979 and YN29 were constructed and sequenced. Over 100 million raw reads were generated in each library (among 100.9 to 104.7 million raw reads in each library). About $76 \%$ to $85 \%$ reads were successfully mapped to wheat genome (TGAC v1.0). Find_circ software was employed to detect head-to-tail splicing (back-spliced) of the remaining 15-24\% unmapped RNA-seq reads. The number of circRNAs identified in each sample ranged from 285 to 478. Among them, more than 70\% of circRNAs are exonic circRNAs (Fig. 2a). The proportion of intronic circRNAs is the lowest, no more than $6.5 \%$ in each sample. The proportion of intergenic circRNAs is between the two, accounting for 18.7-24.9\% (Fig. 2a).

\section{Identification of differentially expressed circRNAs}

We compared the circRNAs expression profiles in the long root varieties (XN979 and LH9) and the short root variety (YN29), and obtained DECs between them. In the YN29-LH9 comparison, 33 circRNAs were identified as DECs ( $\mathrm{p}$ value $\leq 0.05$ along with $\mid \log _{2}$ (foldchange) $\mid \geq 1$ ) (Fig. 2b; Additional file 2: Table S2). Among them, 18 circRNAs were up-regulated and 15 were down-regulated in YN29 compared with LH9 (Fig. 2c). We totally identified 22 DECs in the one to one comparison between YN29 and XN979 (Fig. 2b; Additional file 3: Table S3). Among them, 15 circRNAs showed up-regulation and seven 


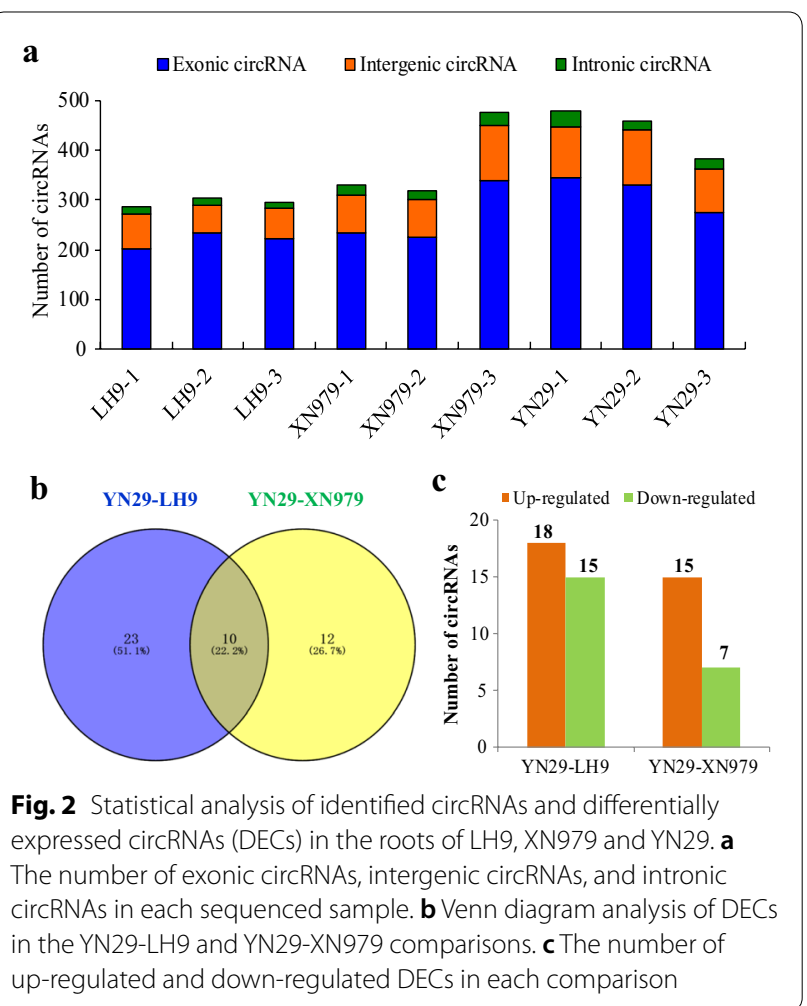

circRNAs exhibited down-regulation in YN29 compared with XN979 (Fig. 2c). Among the circRNAs identified above, ten circRNAs existed in both the YN29-LH9 comparison and the YN29-XN979 comparison (Fig. 2b; Table 1). Twenty-three DECs were specifically found in the YN29-LH9 comparison and 12 DECs were specifically found in the YN29-XN979 comparison (Fig. 2b). Since the roots of both LH9 and XN979 are significantly larger and deeper than those of YN29 (Fig. 1c), the ten
DECs coexisting in the two comparisons are highly likely to be involved in the regulation of wheat root length.

\section{Real-time PCR verification of DECs}

Five randomly selected DECs were selected for data verification of RNA-seq by using real-time PCR technology. Results showed that the expression levels of the five DECs detected using real-time PCR technology matched well with the results of RNA-seq (Fig. 3), indicating that the results of RNA-seq are reliable.

\section{The regulation of miRNAs levels by circRNAs}

It has been reported that circRNAs can act as ceRNAs (competitive endogenous RNA) of miRNAs, inhibiting their binding to mRNA molecules, thereby regulating gene expression [21, 44]. To detect whether the ten DECs coexisted in both comparisons can perform the function, psRNATarget software was employed to predict potential miRNA binding sites of the circRNAs. Results showed that three out of the ten DECs were predicted to have two to nine corresponding miRNAs binding sites (Table 1). CircRNA23 have putative miRNA binding sites for taemiR1134 and tae-miR5085. CircRNA3645 have putative miRNA binding sites for tae-miR1133, tae-miR1137a and tae-miR9773. According to the psRNATarget-based prediction, circRNA2473 can bind to nine different miRNAs. To investigate whether these circRNAs regulate the expression of the corresponding miRNAs, eight potential circRNA-binding miRNAs were selected to check their expression levels in the long root plants (LH9 and XN979) and the short root plants (YN29). Results showed that six of them exhibited significant differences between the long root plants (LH9 and XN979) and the short root plants (YN29) (Fig. 4).

Table 1 CircRNAs involved in the regulation of wheat root length

\begin{tabular}{|c|c|c|c|}
\hline Name & Position & Chr & Corresponding miRNAs \\
\hline $\operatorname{circRNA23}$ & 1B:60935-76949 & TGACv1_scaffold_049914_1BS & tae-miR1134; tae-miR5085 \\
\hline circRNA2473 & $6 B: 456-24596$ & TGACv1_scaffold_500983_6BL & $\begin{array}{l}\text { tae-miR164; tae-miR1134; tae-miR1122b-3p; tae-miR1125; } \\
\text { tae-miR1118; tae-miR1137a; tae-miR1122a; tae-miR1133; } \\
\text { tae-miR9773 }\end{array}$ \\
\hline circRNA3645 & $U: 124270-137099$ & TGACv1_scaffold_641408_U & tae-miR1133; tae-miR1137a; tae-miR9773 \\
\hline circRNA1237 & 1A:102008-120691 & TGACv1_scaffold_000490_1AL & None \\
\hline circRNA3004 & 1B:69161-71982 & TGACv1_scaffold_049372_1BS & None \\
\hline circRNA2496 & 1D:61364-61592 & TGACv1_scaffold_080514_1DS & None \\
\hline circRNA3197 & 2B:52297-53454 & TGACv1_scaffold_147474_2BS & None \\
\hline circRNA832 & 2D:14143-15315 & TGACv1_scaffold_178493_2DS & None \\
\hline circRNA198 & $3 A: 8606-9403$ & TGACv1_scaffold_197368_3AL & None \\
\hline circRNA956 & $7 A: 20092-74718$ & TGACv1_scaffold_569274_7AS & None \\
\hline
\end{tabular}


a

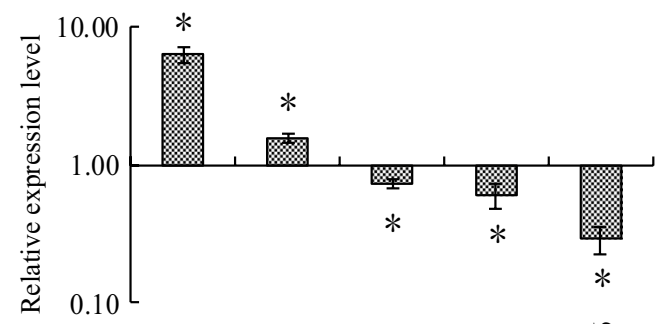

b

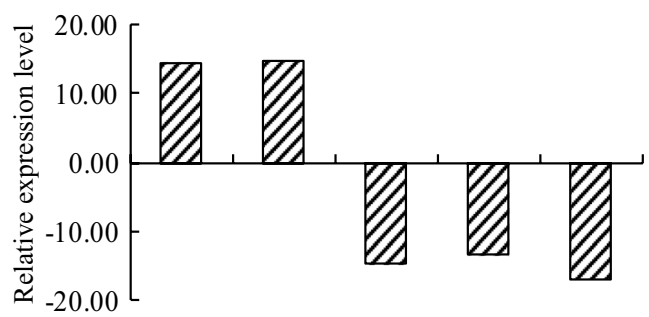

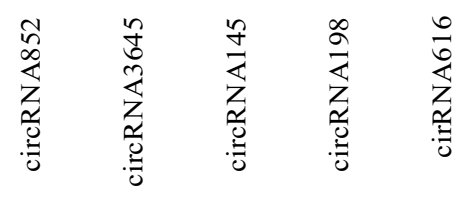

Fig. 3 Relative expression analysis of differentially expressed circRNAs (DECs). a The results of relative expression analysis of circRNAs by using real-time PCR technology $\left({ }^{*} p<0.05\right)$. b The results of relative expression analysis of circRNAs by using RNA-sequence technology

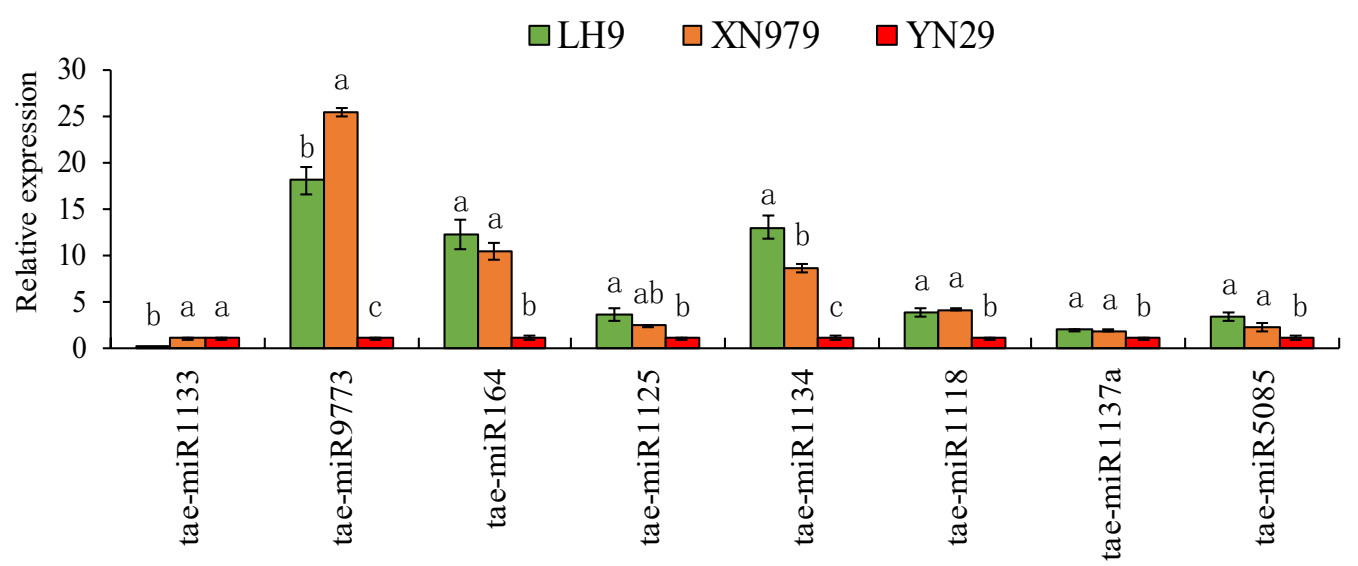

Fig. 4 Relative expression analysis of potential binding miRNAs of the DECs in the roots of LH9, XN979 and YN29. Significant difference at $p<0.05$ is indicated by different letters above the columns

\section{Discussion}

Deeper rooting is beneficial for plant production and survival under water and nitrogen deficient conditions [48-50]. Therefore, genetic improvement of root traits is essential for improving crop nutrient and water use efficiencies. Recent studies have shown that circRNAs are widespread in plants and play important roles in regulating plant growth and development, and stress tolerance [23-41]. However, whether circRNAs, as a new type of non-coding RNA molecules, participate in the regulation of root growth is not clear. Here, an integrated comparative root transcriptomic study of long root plants (LH9 and XN979) and short root plants (YN29) was conducted to explore this question. The number of circRNAs identified in each sample ranged from 285 to 478 . More than $70 \%$ circRNAs belong to exonic circRNAs category. The intronic circRNAs only account for 3.9-6.5\% of the total number of circRNAs in different samples (Fig. 2a). The proportion of intronic circRNAs is similar to the results of previous reports in Arabidopsis (3.8\% intronic circRNAs), tomato (3.6\%) and wheat (less than 6.5\%) $[16,19$, 41]. In the YN29-LH9 comparison and YN29-XN979 comparison, 33 and 22 DECs were identified, respectively (Fig. 2b). In total, ten circRNAs existed in both the YN29LH9 comparison and the YN29-XN979 comparison. As has been mentioned above, both the roots of LH9 and XN979 were significantly larger and deeper than those of YN29 (Fig. 1c), therefore, the ten coexisted DECs in both comparisons should play more critical roles in the regulation of root length.

Previous studies in animal and human have confirmed that circRNAs can act as miRNA sponges to capture 
miRNAs from their target genes via ceRNA (competing endogenous RNAs) networks [51, 52]. Similar reports have recently been made in plants [26, 34, 38, 41]. To reveal whether the ten DECs can target miRNAs and participate in transcriptional regulation of genes, psRNATarget software was used to identify potential miRNA binding sites. As expected, three of the ten circRNAs had two to nine putative miRNA-binding sites (Table 1), which was consist with another study in wheat [38]. Moreover, the results of real-time PCR analysis showed that the expression levels of six out of the eight investigated potential binding miRNAs exhibited significant differences between the long root plants (LH9 and XN979) and the short root plants (YN29) (Fig. 4). Therefore, the expression levels of these miRNAs were correlated with their corresponding circRNAs. These results indicate that the three circRNAs may bind to the target miRNAs and modulate the transcript levels of these miRNAs. Interestingly, it has been reported that some putative target miRNAs are involved in the regulation of plant growth and development [53-57]. For example, miR164 has been reported controlling root development in Arabidopsis, maize and potato [53-55]. In Arabidopsis, one of miR164 target genes is $N A C 1$, which transduces auxin signals for lateral root emergence [58, 59]. In mir164 mutants, plants express less miR164 and more NAC1 mRNA, and produced more lateral roots. Moreover, the mutant phenotypes can be complemented by expression miR164a and miR164b genomic sequences [53]. Besides, the target genes of the potential circRNA binding miRNAs such as miR1122, miR1125, miR1134 and miR1133 are also involved in plant growth, development, metabolism and stress response $[56,57]$. Therefore, these circRNAs may regulate wheat root length by modulating target miRNAs levels. We also noticed that different circRNAs may have the same miRNA binding site, and one circRNA may have several corresponding miRNA binding sites (Table 1). Since the mechanism of how circRNAs regulate their target miRNA are not clear in plants so far and these miRNA binding sites are predicted by psRNATarget software, whether circRNA can bind to each of them still requires further experimental validation. Furthermore, how do the circRNAs that do not have miRNAs binding sites work? What are the mechanisms by which different circRNAs coordinately regulate the length of wheat roots? There is still a lot of work to be done to clarify the regulatory roles of these circRNAs.

\section{Conclusions}

Our study revealed that the expression levels of some circRNAs in roots exhibited significant differences between the long root plants (LH9 and XN979) and the short root plants (YN29). Ten DECs involved in the regulation of wheat root length were successfully identified, three of which have potential miRNAs binding sites. The expression levels of putative circRNA-binding miRNAs were correlated with their corresponding circRNAs. These results provide new clues for investigating the functions of circRNAs in the regulation of wheat root length.

\section{Additional files}

Additional file 1: Table S1. Primers used for real-time PCR.

Additional file 2: Table S2. List of differentially expressed circRNAs (DECs) in the YN29-LH9 comparison.

Additional file 3: Table S3. List of differentially expressed circRNAs (DECs) in the YN29-XN979 comparison.

\section{Abbreviations}

BR: brassinosteroid; ceRNA: competing endogenous RNA; circRNAs: circular RNAs; DECs: differentially expressed circRNAs; MRL: maximum root length; rRNA: ribosomal RNAs; TRL: total root length.

\section{Authors' contributions}

YX wrote the paper. YR performed most of the experiments; $Y X$ took part in partial work of this research; YR, DC and TL conceived and designed the experiments; DC gave many helpful suggestions on the writing. All authors read and approved the final manuscript.

\section{Author details \\ ${ }^{1}$ College of Agronomy, Henan Agricultural University, Zhengzhou 450002, China. ${ }^{2}$ State Key Laboratory of Wheat and Maize Crop Science, Henan Agricultural University, Zhengzhou 450002, China. ${ }^{3}$ Collaborative Innova- tion Center of Henan Grain Crops, Henan Agricultural University, Zheng- zhou 450002, China. ${ }^{4}$ College of life science, Shangqiu Normal University, Shangqiu 476000, China.}

\section{Acknowledgements}

We thank Huifang Yue for her help in root phenotypic analysis and data processing.

\section{Competing interests}

The authors declare that they have no competing interests.

\section{Availability of data and materials}

The datasets used and/or analysed during the current study are available from the corresponding author on reasonable request.

\section{Consent for publication}

Not applicable.

\section{Ethics approval and consent to participate}

Not applicable.

\section{Funding}

This work was supported by the National Natural Science Foundation of China (31572197), the National Natural Science Foundation of China (31201678), the Natural Science Foundation of Henan province (162300410133) and the State Key Laboratory Program (PCCE-KF-2017-04).

\section{Publisher's Note}

Springer Nature remains neutral with regard to jurisdictional claims in published maps and institutional affiliations. 
Received: 21 January 2019 Accepted: 29 March 2019

Published online: 04 April 2019

\section{References}

1. de Dorlodot S, Forster B, Pagès L, Price A, Tuberosa R, Draye X. Root system architecture: opportunities and constraints for genetic improvement of crops. Trends Plant Sci. 2007;12:474-81.

2. Lynch J. Roots of the second green revolution. Aust J Bot. 2007;55:493-512.

3. Den Herder G, Van Isterdael G, Beeckman T, De Smet I. The roots of a new green revolution. Trends Plant Sci. 2010;15:600-7.

4. White PJ, George TS, Gregory PJ, Bengough AG, Hallett PD, McKenzie BM. Matching roots to their environment. Ann Bot. 2013;112:207-22.

5. Yu P, Li XX, White PJ, Li CJ. A large and deep root system underlies high nitrogen-use efficiency in maize production. PLOS ONE. 2015:10:e0126293.

6. Pacifici E, Polverari L, Sabatini S. Plant hormone crosstalk: the pivot of root growth. J Exp Bot. 2015;66:1113-21.

7. Dello loio R, Nakamura K, Moubayidin L, Perilli S, Taniguchi M, Morita MT, Aoyama T, Costantino P, Sabatini S. A genetic frame work for the control of cell division and differentiation in the root meristem. Science. 2008:322:1380-4

8. Vragovic K, Sela A, Friedlander-Shani L, Fridman Y, Hacham Y, Holland N, Bartom E, Mockler TC, Savaldi-Goldstein S. Translatome analyses capture of opposing tissue-specific brassinosteroid signals orchestrating root meristem differentiation. Proc Natl Acad Sci USA. 2015;112:923-8.

9. Li J, Nagpal P, Vitart V, McMorris TC, Chory J. A role for brassinosteroids in light-dependent development of Arabidopsis. Science. 1996;272:398-401.

10. Müssig C, Shin GH, Altmann T. Brassinosteroids promote root growth in Arabidopsis. Plant Physiol. 2003;133:1261-71

11. Roddick JG, Rijnenberg AL, Ikekawa N. Developmental effects of 24-epibrassinolide in excised roots of tomato grown in vitro. Physiol Plant. 1993:87:453-8.

12. Clouse SD, Langford M, McMorris TC. A brassinosteroid-insensitive mutant in Arabidopsis thaliana exhibits multiple defects in growth and development. Plant Physiol. 1996;111:671-8.

13. He X, Fang JJ, Li JJ, Qu BY, Ren YZ, Ma WY, Zhao X, Li B, Wang DW, Li ZS, et al. A genotypic difference in primary root length is associated with the inhibitory role of transforming growth factor-beta receptor-interacting protein-1 on root meristem size in wheat. Plant J. 2014;77:931-43.

14. De Tullio MC, Jiang K, Feldman $\sqcup$. Redox regulation of root apical meristem organization: connecting root development to its environment. Plant Physiol Biochem. 2010;48:328-36.

15. Xia XJ, Chen Z, Yu JQ. ROS mediate brassinosteroids-induced plant stress responses. Plant Signal Behav. 2010;5:532-4.

16. Lasda E, Parker R. Circular RNAs: diversity of form and function. RNA. 2014;20:1829-42.

17. Chen LL, Yang L. Regulation of circRNA biogenesis. RNA Biol. 2015:12:381-8.

18. Salzman J, Gawad C, Wang PL, Lacayo N, Brown PO. Circular RNAs are the predominant transcript isoform from hundreds of human genes in diverse cell types. PLOS ONE. 2012;7:e30733.

19. JeckWR, Sharpless NE. Detecting and characterizing circular RNAs. Nat Biotechnol. 2014;32:453-61.

20. Westholm JO, Miura P, Olson S, Shenker S, Joseph B, Sanfilippo P, Celniker SE, Graveley BR, Lai EC. Genome-wide analysis of Drosophila circular RNAs reveals their structural and sequence properties and age-dependent neural accumulation. Cell Rep. 2014;9:1966-80.

21. Fan $X Y$, Zhang $X N$, Wu XL, Guo HS, Hu YQ, Tang FC, Huang YY. Single-cell RNA-seq transcriptome analysis of linear and circular RNAs in mouse preimplantation embryos. Genome Biol. 2015;16:148.

22. Sablok G, Zhao H, Sun X. Plant Circular RNAs (circRNAs): transcriptional regulation beyond miRNAs in plants. Mol. Plant. 2016;9:192-4.

23. Pan T, Sun XQ, Liu YX, Li H, Deng GB, Lin HH, Wang SH. Heat stress alters genome-wide profiles of circular RNAs in Arabidopsis. Plant Mol Biol. 2018:96:217-29.

24. Liu TF, Zhang L, Chen G, Shi TL. Identifying and characterizing the circular RNAs during the lifespan of Arabidopsis leaves. Front Plant Sci. 2017:8:1278.
25. Ye CY, Chen L, Liu C, Zhu QH, Fan L. Widespread noncoding circular RNAs in plants. New Phytol. 2015;208:88-95.

26. Chen G, Cui JW, Wang L, Zhu YF, Lu ZG, Jin B. Genome-wide identification of circular RNAs in Arabidopsis thaliana. Front Plant Sci. 2017:8:1678.

27. Meng X, Zhang P, Chen Q, Wang J, Chen M. Identification and characterization of ncRNA-associated ceRNA networks in Arabidopsis leaf development. BMC Genomics. 2018;19:607.

28. Zhao W, Cheng YH, Zhang C, You QB, Shen XJ, Guo W, Jiao YQ. Genomewide identification and characterization of circular RNAs by high throughput sequencing in soybean. Sci. Rep. 2017;7:5636.

29. Chen L, Ding X, Zhang H, He T, Li Y, Wang T, Li X, Jin L, Song Q, Yang S, et al. Comparative analysis of circular RNAs between soybean cytoplasmic male-sterile line NJCMS1A and its maintainer NJCMS1B by highthroughput sequencing. BMC Genomics. 2018;19:663.

30. Lu TT, Cui LL, Zhou Y, Zhu CR, Fan DL, Gong H, Zhao Q, Zhou CC, Zhao Z, Lu DF, et al. Transcriptome-wide investigation of circular RNAs in rice. RNA. 2015;21:2076-87.

31. Chen L, Zhang P, Fan Y, Lu Q, Li Q, Yan J, Muehlbauer GJ, Schnable PS, Dai M, Li L. Circular RNAs mediated by transposons are associated with transcriptomic and phenotypic variation in maize. New Phytol. 2017;217:1292-306

32. Ghorbani A, Izadpanah K, Peters JR, Dietzgen RG, Mitter N. Detection and profiling of circular RNAs in uninfected and maize Iranian mosaic virusinfected maize. Plant Sci. 2018:274:402-9.

33. Tang B, Hao Z, Zhu Y, Zhang H, Li G. Genome-wide identification and functional analysis of circRNAs in Zea mays. PLoS ONE. 2018;13:e0202375.

34. Zuo J, Wang Q, Zhu B, Luo Y, Gao L. Deciphering the roles of circRNAs on chilling injury in tomato. Biochem Biophys Res Commun. 2016;479:132-8.

35. Wang J, Yang Y, Jin L, Ling X, Liu T, Chen T, Ji Y, Yu W, Zhang B. Re-analysis of long non-coding RNAs and prediction of circRNAs reveal their novel roles in susceptible tomato following TYLCV infection. BMC Plant Biol. 2018;18:104.

36. Darbani B, Noeparvar S, Borg S. Identification of circular RNAs from the parental genes involved in multiple aspects of cellular metabolism in barley. Front. Plant Sci. 2016;7:776.

37. Tong W, Yu J, Hou Y, Li F, Zhou Q, Wei C, Bennetzen JL. Circular RNA architecture and differentiation during leaf bud to young leaf development in tea (Camellia sinensis). Planta. 2018;248:1417-29.

38. Zhao T, Wang L, Li S, Xu M, Guan X, Zhou B. Characterization of conserved circular RNA in polyploid Gossypium species and their ancestors. FEBS Lett. 2017;591:3660-9.

39. Xiang L, Cai C, Cheng J, Wang L, Wu C, Shi Y, Luo J, He L, Deng Y, Zhang X, et al. Identification of circularRNAs and their targets in Gossypium under Verticillium wilt stress based on RNA-seq. PeerJ. 2018;6:e4500.

40. Wang YX, Yang M, Wei SM, Qin FJ, Zhao HJ, Suo B. Identification of circular RNAs and their targets in leaves of Triticum aestivum L. under dehydration stress. Front. Plant Sci. 2017;7:2024.

41. Ren Y, Yue H, Li L, Xu Y, Wang Z, Xin Z, Lin T. Identification and characterization of circRNAs involved in the regulation of low nitrogen-promotedroot growth in hexaploid wheat. Biol Res. 2018;51:43.

42. Ren YZ, He X, Liu DC, Li JJ, Zhao XQ, Li B, Tong YP, Zhang AM, Li ZS. Major quantitative trait loci for seminal root morphology of wheat seedlings. Mol Breeding. 2012:30:139-48.

43. Zadoks JC, Chang TT, Konzak CF. Decimal code for the growth stages of cereals. Weed Res. 1974;4:415-21.

44. Memczak S, Jens M, Elefsinioti A, Torti F, Krueger J, Rybak A, Maier L, Mackowiak SD, Gregersen LH, Munschauer M, et al. Circular RNAs are a large class of animal RNAs with regulatory potency. Nature. 2013;495:333-8.

45. Zhou L, Chen JH, Li ZZ, Li XX, Hu XD, Huang Y, Zhao XK, Liang CZ, Wang $Y$, Sun $L$, et al. Integrated profiling of microRNAs and mRNAs: microRNAs located on Xq27.3 associate with clear cell renal cell carcinoma. PloS ONE. 2010;5:e15224.

46. Anders S, Huber W. Differential expression analysis for sequence count data. Genome Biol. 2010;11:R106.

47. Dai X, Zhao PX. psRNATarget: a plant small RNA target analysis server. Nucleic Acids Res. 2011;39:W155.

48. Uga Y, Sugimoto K, Ogawa S, Rane J, Ishitani M, Hara N, Kitomi Y, Inukai Y, Ono K, Kanno N, et al. Control of root system architecture by DEEPER ROOTING 1 increases rice yield under drought conditions. Nat Genet. 2013:45:1097-102. 
49. Comas LH, Becker SR, Cruz VMV, Byrne PF, Dierig DA. Root traits contributing to plant productivity under drought. Front Plant Sci. 2013;4:442.

50. Koevoets IT, Venema JH, Elzenga JT, Testerink C. Roots withstanding their environment: exploiting root system architecture responses to abiotic stress to improve crop tolerance. Front Plant Sci. 2016;31:1335.

51. Hansen TB, Jensen TI, Clausen BH, Bramsen JB, Finsen B, Damgaard CK, Kjems J. Natural RNA circles function as efficient microRNA sponges. Nature. 2013:495:384-8.

52. Zhang S, Zhu D, Li H, Li H, Feng C, Zhang W. Characterization of circRNAassociated-ceRNA networks in a senescence-accelerated mouse prone 8 brain. Mol Ther. 2017;25:2053-61.

53. Guo HS, Xie Q, Fei JF, Chua NH. MicroRNA directs mRNA cleavage of the transcription factor NAC1 to downregulate auxin signals for Arabidopsis lateral root development. Plant Cell. 2005;17:1376-86.

54. Li J, Guo GH, Guo WW, Guo GG, Tong D, Ni ZF, Sun QX, Yao YY. miRNA164directed cleavage of $Z m N A C 1$ confers lateral root development in maize (Zea mays L.). BMC Plant Biol. 2012;12:220.
55. Zhang L, Yao L, Zhang N, Yang J, Zhu X, Tang X, Calderón-Urrea A, Si H. Lateral root development in potato is mediated by stu-mi164 regulation of NAC transcription factor. Front Plant Sci. 2018;9:383.

56. Pandey B, Gupta OP, Pandey DM, Sharma I, Sharma P. Identification of new stress-induced microRNA and their targets in wheat using computational approach. Plant Signal Behav. 2013;8:e23932.

57. Zhou L, Liu Y, Liu Z, Kong D, Duan M, Luo L. Genome-wide identification and analysis of drought-responsive microRNAs in Oryza sativa. J Exp Bot. 2010;61:4157-68.

58. Rhoades MW, Reinhart BJ, Lim LP, Burge CB, Bartel B, Bartel DP. Prediction of plant microRNA targets. Cell. 2002;110:513-20.

59. Xie Q, Guo HS, Dallman G, Fang S, Weissman AM, Chua NH. SINAT5 promotes ubiquitin-related degradation of NAC1 to attenuate auxin signals. Nature. 2002;419:167-70.
Ready to submit your research? Choose BMC and benefit from:

- fast, convenient online submission

- thorough peer review by experienced researchers in your field

- rapid publication on acceptance

- support for research data, including large and complex data types

- gold Open Access which fosters wider collaboration and increased citations

- maximum visibility for your research: over $100 \mathrm{M}$ website views per year

At BMC, research is always in progress.

Learn more biomedcentral.com/submissions 